\title{
Contraception Choice and Compliance Among Mothers with Opioid Addiction: Identifying Barriers to Care
}

\author{
Robert C. Bowers, MD'Catherine Waldron ${ }^{1}$, Hayley Weese' ${ }^{1}$, Jennie Yoost MD, MSc ${ }^{1}$, Amy Roy, MD'1
}

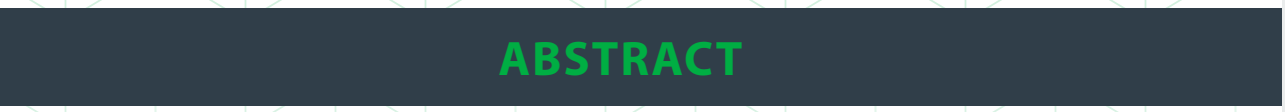

West Virginia leads the nation in substance abuse deaths per capita. This epidemic has given rise to a novel population of high-risk, obstetric patients with opiate abuse. Appropriate education, counseling, and implementation of contraception are of extreme importance among this population, due to the risks of unintended pregnancy. This study was a retrospective chart review to determine compliance and uptake of contraceptive methods by patients in a maternal buprenorphine maintenance program prior to and following implementation of standardized contraception education and Medicaid coverage of immediate postpartum long-acting reversible contraceptives (LARC). Rates of planned contraception and rates of actual method initiation were compared between two groups representing two distinct time periods before and after the intervention. Rates of highly effective contraception options were noted to significantly increase following dedicated education and Medicaid coverage $(p=0.04)$. Patients receiving no contraception at all significantly decreased following these interventions $(p=0.003)$. Patients desiring surgical sterilization continued to encounter barriers to care as the rate of actual sterilization to desired sterilization remained low.
KEYWORDS

Substance Abuse, Maternal, Compliance, Contraception

\section{INTRODUCTION}

For two consecutive years in 2017 and 2018, West Virginia had the highest number of overdose deaths per capita in the United States at 57.8 and 51.5 per 100,000 , respectively. ${ }^{1}$ Among the sizable opioid abusing population of West Virginia, the obstetric population has emerged as a high-risk cohort necessitating specialized care during the antepartum and postpartum periods. An important aspect of their obstetric care is postpartum contraception management. Long-acting reversible contraceptives (LARC), such as intrauterine devices (IUDs) and implants, are the most effective forms of reversible contraception and do not require user effort to be effective. ${ }^{2}$ Given that the rate of unintended pregnancy is estimated at $86 \%$ among opioid-dependent women, the use of effective contraception for this patient population is paramount. ${ }^{3}$ LARC methods are the least expensive forms of reversible contraception over five years, 4 exhibit normal returns of fertility after discontinuation, have a low rate of complications, and have high rates of patient satisfaction. ${ }^{5}$ LARC methods would benefit these high-risk pregnant women with opiate abuse.

This population faces several unique barriers to
Author affiliations are listed at the end of this article.

Corresponding Author: Robert C Bowers, MD Marshall University Joan C. Edwards School of Medicine bowers34@marshall.edu appropriate contraception management including compliance, knowledge deficits, and access to care. It has been previously established that this patient population has very poor compliance to postpartum visits, a time when most contraceptive counseling and implementation occur. ${ }^{6}$ Additionally, state law currently mandates that written consent must be obtained 30 days in advance of any routine delivery or 72 hours in advance of a preterm delivery for a sterilization procedure to be covered by Medicaid"; this stipulation can prevent women with undesired fertility from pursuing sterilization due to concerns for out-of-pocket cost. According to the Contraceptive CHOICE project, in the absence of barriers such as knowledge and cost, the rate of initiation of LARC was higher than other contraceptive methods. Accessible, affordable, easy-to-use contraception, such as LARC, should be a major focus when counseling these highrisk patients. ${ }^{8}$

The Maternal Addiction and Recovery Center (MARC) program at our institution provides specialty care for pregnant women with substance abuse. In a past study looking at a cohort of women from 2010-2014, planned and actual contraception were evaluated, along with compliance with a postpartum visit. This 
Table 1: Baseline Demographic Information

\begin{tabular}{|c|c|c|c|}
\hline & Cohort $1(n=61)$ & Cohort $2(n=173)$ & p value \\
\hline Age & 26.7 (SD 5.1) & 28.7 (SD 4.5) & 0.003 \\
\hline $\begin{array}{c}\text { Race }(\%) \\
\text { White } \\
\text { Black } \\
\text { Other }\end{array}$ & $\begin{array}{c}61(100) \\
0 \\
0\end{array}$ & $\begin{array}{c}170(98.3) \\
2(1.2) \\
1(0.6)\end{array}$ & 0.58 \\
\hline $\begin{array}{c}\text { Delivery }(\%) \\
\text { Vaginal } \\
\text { Cesarean }\end{array}$ & $\begin{array}{l}40(65.6) \\
21(34.4)\end{array}$ & $\begin{array}{c}101(58.4) \\
72(41.6)\end{array}$ & 0.32 \\
\hline Smoker $(\%)$ & $54(88.5)$ & $155(89.6)$ & 0.82 \\
\hline $\begin{array}{c}\text { Insurance }(\%) \\
\text { Medicaid } \\
\text { Private } \\
\text { Self Pay }\end{array}$ & $\begin{array}{c}40(92.5)^{*} \\
2(5.2) \\
0\end{array}$ & $\begin{array}{c}160(92.5) \\
9(5.2) \\
2(1.2)\end{array}$ & 0.79 \\
\hline
\end{tabular}

Table 2: Postpartum Compliance and Rapid Repeat Pregnancy

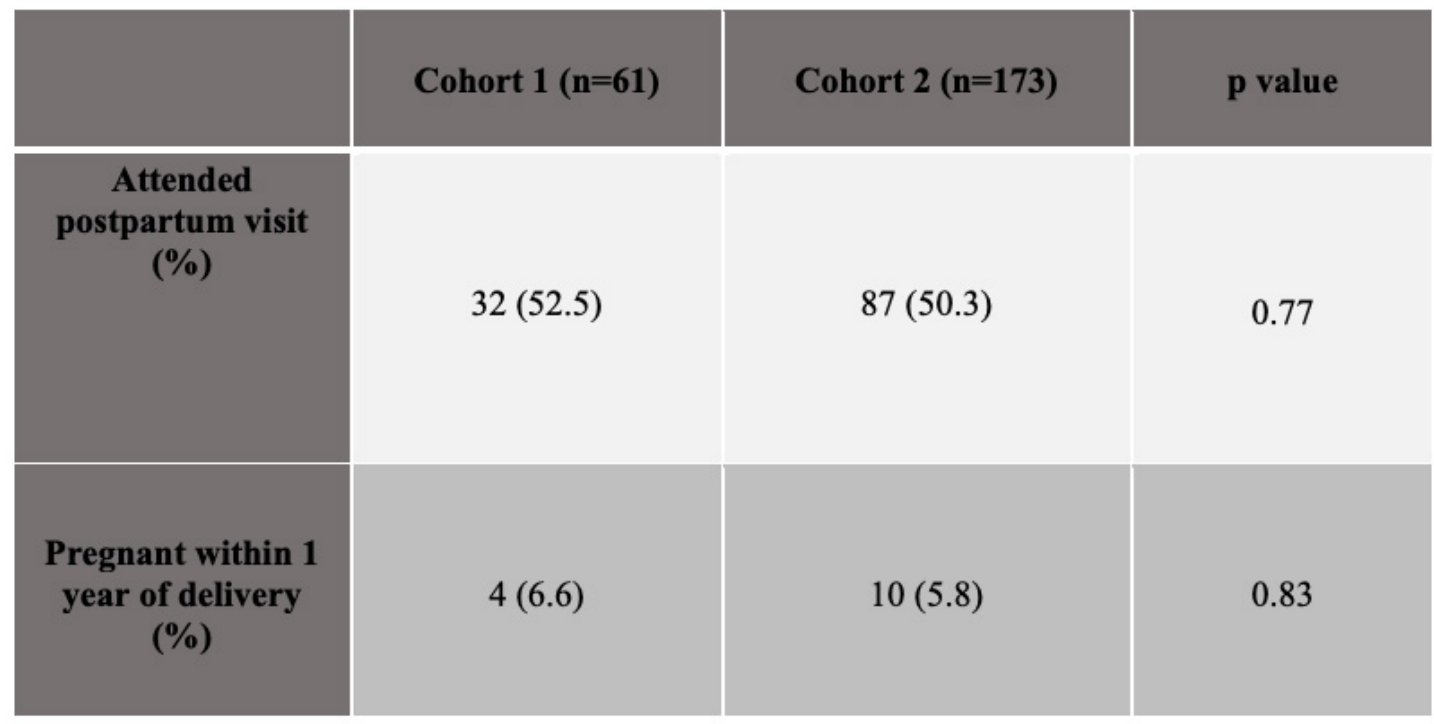

study demonstrated poor LARC uptake among women with substance abuse, poor compliance with pre-pregnancy desired contraception choices, an increased rate of changing to lower efficacy contraception options, and poor compliance with postpartum visits. ${ }^{9}$ Since this study, a standardized contraception counseling intervention was implemented within the MARC program. This intervention provided education to MARC patients about all birth control methods during their antenatal care. Additionally, in 2016, West Virginia Medicaid implemented coverage for immediate postpartum (IPP) LARC placement. IPP LARCs are done immediately after delivery during the inpatient stay. This practice is thought to reduce barriers and improve compliance with LARC methods.

The purpose of this study was to determine if contraception education provision and IPP LARC access improved contraception usage among pregnant women with opiate abuse. Furthermore, it was the aim of this study to review all contraception choices and successful initiation of those choices among this 
Table 3: Antepartum Planned Contraception

\begin{tabular}{|c|c|c|c|}
\hline & Cohort $1(\mathbf{n}=\mathbf{6 1})$ & Cohort 2(n=173) & p value \\
\hline None/Undecided & $23(37.7)$ & $33(19.1)$ & .003 \\
\hline Tubal ligation & $23(37.7)$ & $77(44.5)$ & 0.36 \\
\hline IUD & $4(6.6)$ & $27(15.6)$ & .07 \\
\hline Implant & $2(3.3)$ & $18(10.4)$ & .08 \\
\hline DMPA & $5(8.2)$ & $12(6.9)$ & .74 \\
\hline Pill/patch/ring & $4(6.6)$ & $6(3.5)$ & .31 \\
\hline
\end{tabular}

Table 4: Actual Postpartum Contraception

\begin{tabular}{|c|c|c|c|}
\hline & Cohort 1(n=61) & Cohort 2 (n=173) & p value \\
\hline None/Undecided & $30(49.2)$ & $67(38.7)$ & .15 \\
\hline Tubal ligation & $16(26.2)$ & $47(27.2)$ & .88 \\
\hline ILD & $2(3.3)$ & $17(9.8)$ & .11 \\
\hline Implant & $0(0)$ & $19(11)$ & .007 \\
\hline DMPA & $13(21.3)$ & $23(13.3)$ & .14 \\
\hline Pill/patch/ring & $8(13.1)$ & $7(4)$ & .013 \\
\hline
\end{tabular}

high-risk obstetric population.

\section{METHODS}

This study was a retrospective chart review including patients enrolled in the MARC program. We defined the population prior to implementation of any barrier-to-care interventions as those patients actively enrolled into the MARC program for their obstetric care from 1/2010-12/2014 (cohort 1), and the population after implementation of interventions as those patients actively enrolled into the MARC program for their obstetric care from 1/2016-12/2018 (cohort 2). The first barrier-to-care intervention was the imple- mentation of a dedicated contraception education curriculum. The MARC program has a rotating list of speakers to discuss various topics with the participants. Approximately every 12 weeks, an OBGYN physician conducted an hour-long formal educational session with MARC participants. This session educated MARC patients on all forms of contraception including LARC methods and sterilization methods such as bilateral tubal ligation and vasectomy. The risks, benefits, bleeding patterns, and procedures of each method were reviewed with adequate time for questions and discussion. The second intervention was new legislation providing Medicaid coverage of immediate postpartum LARC methods. 


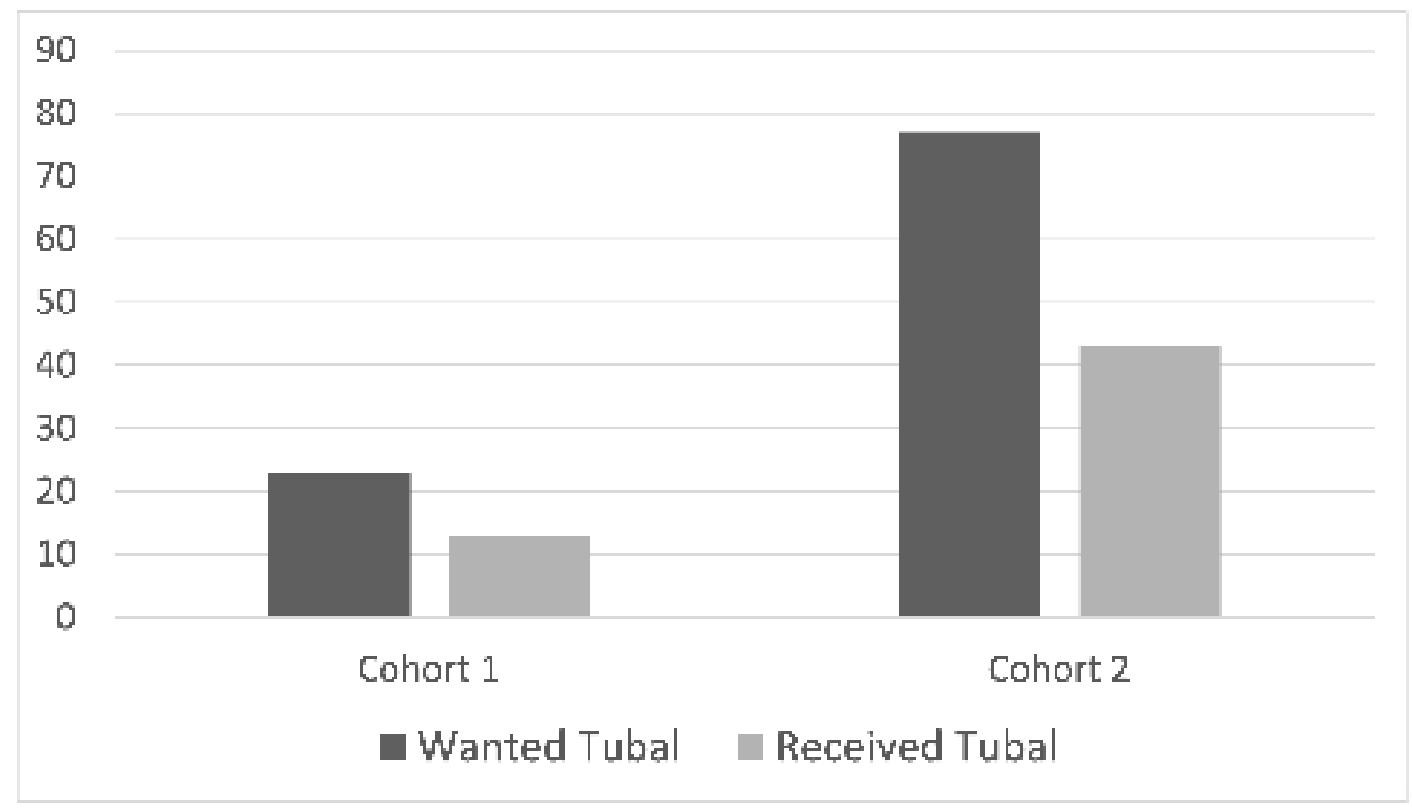

Figure 1: Patients desiring bilateral tubal ligation for contraception vs. patients receiving BTL. Cohort $1-13$ (56.5\%) vs. Cohort $2-43(55.8 \%)(p=.95)$

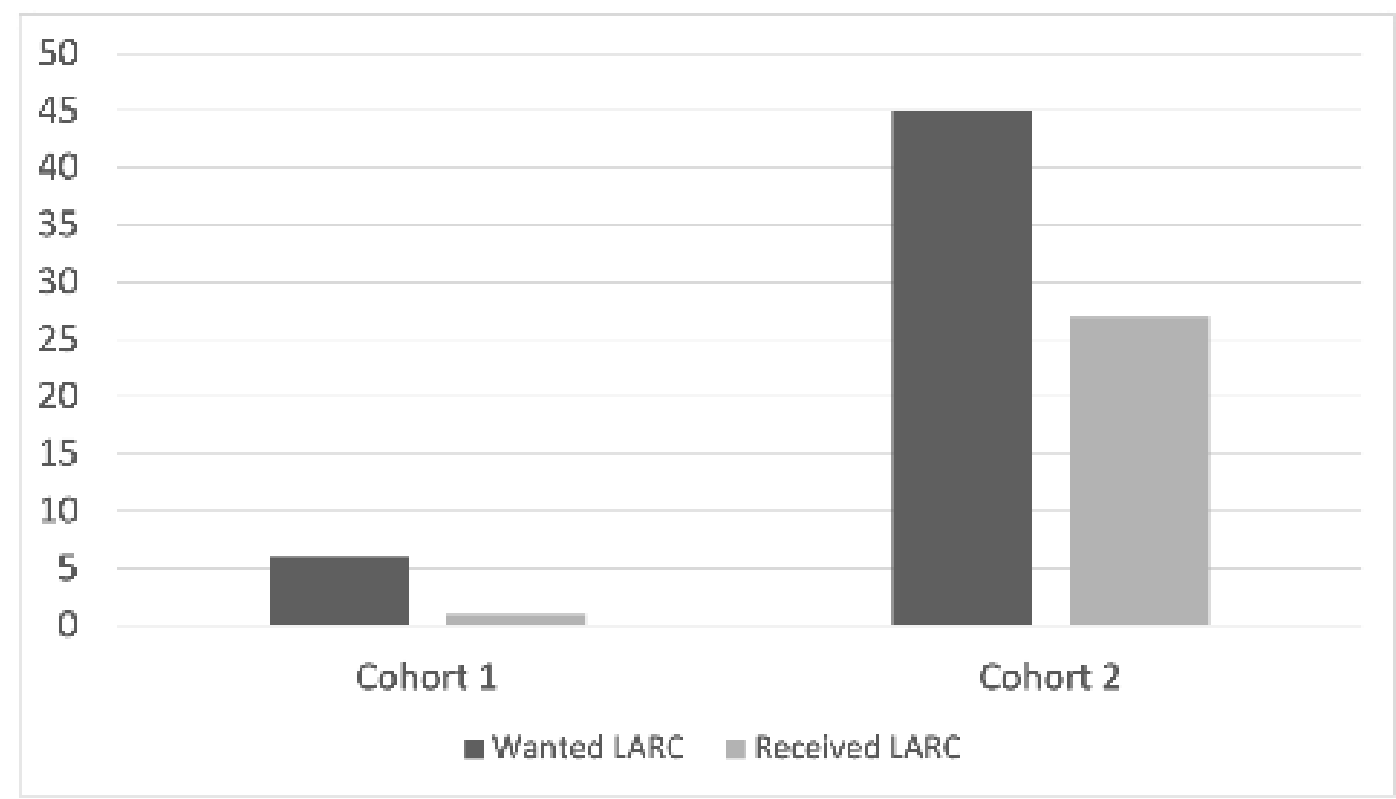

Figure 2: Patients desiring LARC for contraception vs. patients receiving LARC. Cohort 1 - $6(16.7 \%)$ vs. Cohort $2-27(60 \%)(p=.04)$

Data collection concluded prior to 2015 to allow for a full year of follow-up in order to assess the prevalence of short-interval repeat pregnancy. Short-interval repeat pregnancy was defined as pregnancy within a year of delivery. Data were not collected on those patients who were pregnant in 2015 to avoid overlapping the allotted 1 year follow-up period with the implementation of our interventions in 2016. Prenatal charts were reviewed for planned contraception, defined as the contraceptive method documented antenatally that the patient wanted following delivery. Charts were also reviewed for actual contraception, defined as the contraceptive method the patient actually received following delivery. Patients' postpartum notes were used to assess postpartum visit compliance as well as compliance or successful implementation of contraception within six weeks of delivery. Short-interval repeat 
pregnancies were evaluated based on return office visits for a new pregnancy within one year of the antecedent pregnancy. Demographic data were collected from the prenatal record including age, race, gravidity, parity, mode of delivery, smoking status, and history of sexually transmitted diseases. Contraception options were placed into tiers according to efficacy: Tier 1 - LARC and sterilization methods; Tier 2 - hormonal contraceptives including birth control pills, ring, and depot medroxyprogesterone; and Tier 3 - barrier methods including condoms/diaphragms, undecided, or no contraception. Contraceptive use, follow-up, rapid repeat pregnancy rates, and changes in contraceptive efficacy (from what was planned to what was actually given) were compared between groups. Continuous variables were analyzed using a t-test, and categorical variables were analyzed using a chi-square or Fisher's exact test.

\section{RESULTS}

There were 61 patients included in cohort 1 and 173 patients included in cohort 2. Upon comparison of the two groups, the only statistically different demographic value between them was a difference in the mean age (Table 1; cohort $1-26.7$ years, SD 5.1; cohort $2-28.7$ years, SD 4.5; $p=0.003$ ). Of note, there were 19 patients in cohort 1 who had no documentation of any insurance type or self-pay status who were not included in that portion of the demographic data. Rates of postpartum visit compliance within 6 weeks were noted to be 32 (52.5\%) for cohort 1 and 87 (50.3\%) for cohort 2 and did not differ between the cohorts $(p=0.77)$ (Table 2). Rates of short-interval repeat pregnancy also did not differ significantly between the cohorts: 4 (6.6\%) in cohort 1 and 10 $(5.8 \%)$ in cohort $2(p=0.83)$ (Table 2$)$. The rate of "undecided/none" as a planned method of contraception as documented antepartum significantly decreased from $23(37.7 \%)$ in cohort 1 to $33(19.1 \%)$ in cohort $2(p=0.003)$ (Table 3$)$. The "actual contraception initiated" rate for progesterone implant significantly increased from $0(0 \%)$ in cohort 1 to 19 $(11 \%)$ in cohort $2(p=0.007)$ (Table 4). The overall percentage receiving Tier 1 contraception methods increased significantly from 18 (29.5\%) to $83(48.0 \%)$ $(p=0.012)$. Of those patients desiring bilateral tubal ligation for contraception, 13 of the 23 patients in cohort 1 (56.5\%) and 43 of the 77 in cohort 2 (55.8\%) actually received a sterilization procedure $(p=.95)$
(Figure 1). Of the 6 patients who desired LARC antepartum, only one patient received LARC in cohort 1 $(16.7 \%)$ as compared to 27 of the $45(60 \%)$ in cohort $2(p=0.04)$ (Figure 2$)$.

\section{DISCUSSION}

At the start of this study, WV Medicaid initiated reimbursement for immediate postpartum (IPP) LARC provision, effectively ameliorating two of the proposed barriers to care facing substance abuse patients: poor compliance with postpartum follow-up and concern for cost. Prior to 2016, patients desiring LARC were required to attend postpartum visits to obtain a contraceptive implant or IUD. Nearly half of the MARC population (45.9\%), regardless of planned contraception method, received no contraception postpartum in cohort 1. With the implementation of rigorous in-office resident-physician counseling and availability of IPP options, that number decreased to $19.1 \%$ in cohort 2 . After making IPP LARC available, significantly more patients from cohort 2 chose LARC methods antepartum (45\%) and actually implemented LARC methods postpartum ( $n=27,60 \%$ of expected). This finding is in contrast to other studies demonstrating low use of LARCs among opioid-addicted patients from 5-13\%.10,11 Despite improvements in contraception counseling and implementation of planned contraception, significant loss to follow-up among MARC patients persisted between cohorts (52.5\% vs. $50.3 \%$ [ $p=0.77])$. This persistent non-compliance with postpartum visits emphasizes the importance of antepartum contraception counseling, provision of LARC options prior to leaving the hospital, and ensuring those patients with undesired fertility actually receive their sterilization procedures. The vast majority of patients included in this trial were Medicaid recipients (92.5\%) and were therefore eligible for IPP LARC options. Regardless of their antepartum contraception choices, adequate counseling on the safety, efficacy, ease of use, and cost coverage of LARC options should be provided to these patients.

In our study, only $55.8 \%$ of MARC patients in cohort 2 received a bilateral tubal ligation when desired; this is consistent with the rate from cohort 1 (56.5\%). There are multiple proposed reasons for the low rate of surgical sterilization. West Virginia Medicaid requires patients to be over 21 years old, to be med- mds.marshall.edu/mjm

(c) 2021 Marshall Journal of Medicine
Marshall Journal of Medicine Volume 7 Issue 3 
ically competent, and to have signed a state bilateral tubal ligation consent form 30 days in advance but not more than 180 days in advance of delivery. There is an emergent delivery/abdominal surgery clause, however, stating that the time from consent to the time of delivery/surgery must be 72 hours. These stringent additional stipulations may deter patients with undesired fertility from pursuing surgical sterilization. Additionally, operating room availability, surgeon availability, and anesthesia availability often preclude immediate post-delivery tubal ligations which may further contribute to the low rate of completion of desired sterilization procedures.

As a retrospective chart review, the data are limited by accurate medical records, in this instance resident clinic antepartum and postpartum documentation. Further limitations included loss-to-follow-up. Among patients that did not keep postpartum follow-up, it is impossible to determine if they pursued any form of contraception with another provider or went on to have a rapid-repeat pregnancy. A final confounding factor was the introduction and implementation of multiple interventions simultaneously. It is difficult to distinguish which intervention had a greater impact upon patient contraception choice: increased counseling or greater ease of access to care.

We have documented an increase in postnatal LARC uptake and reductions in no-contraception as a planned method of contraception following the introduction of structured prenatal contraception education and concurrent Medicaid coverage for immediate postnatal effective contraception. As numbers and follow-up increase, we speculate that these findings will translate into a reduced number of unintended pregnancies in this vulnerable population. Continued efforts must be made to appropriately educate and counsel the high-risk obstetric population with substance abuse in West Virginia regarding contraception options and availability. It is the responsibility of obstetric providers to continue to educate patients on the safety, efficacy, and ease of use related to LARC methods as these methods become more easily available immediately postpartum. It is also paramount to continue identifying and rectifying barriers keeping high-risk patients from the care they deserve.

\section{AUTHOR AFFILIATION}

1. Marshall University Joan C. Edwards School of Medicine, Huntington, West Virginia

\section{REFERENCES}

1. Opioid Overdose. Centers for Disease Control and Prevention. 2018.

2. Practice Bulletin No. 121: Long-acting reversible contraception: Implants and intrauterine devices. Obstet Gynecol 2011;118(1):184-196.

3. Heil SH, Jones HE, Arria A, et al. Unintended pregnancy in opioid-abusing women. J Subst Abuse Treat 2011;40(2):199-202.

4. Andersson K, Batar I, Rybo G. Return to fertility after removal of a levonorgestrel-releasing intrauterine device and Nova-T. Contraception 1992;46(6):575-584.

5. Trussell JJ, Lalla A, Doan Q, Reyes E, Pingto L, Gricar J. Cost effectiveness of contraceptives in the United States. Contraception 2009;80(2):229-230.

6. Committee Opinion No. 670: Immediate Postpartum Long-Acting Reversible Contraception. Obstet Gynecol 2016;128(2):e32-7.

7. Committee Opinion No. 530: Access to Postpartum Sterilization. American College of Obstetricians and Gynecologists. Obstet Gynecol 2012;120:212-15.

8. Secura GM, Allsworth JE, Madden T, Mullersman JL, Peipert JF. The Contraceptive CHOICE Project: reducing barriers to long-acting reversible contraception. Am J Obstet Gynecol 2010;203(2):115. e111-115.e117.

9. Bowers R, Hatfield-Kresh M, Roy A, Lancaster $D$, Yoost J. Failure of effective contraception in opioid addicted mothers: a disparity in planned and actual usage. Marshall Journal of Medicine 2019;5(1):7.

10. Committee Opinion No. 666: Optimizing Postpartum Care. Obstet Gynecol 2016;127(6):e187-192.

11. Winner B, Peipert JF, Zhao Q, et al. Effectiveness of Long-Acting Reversible Contraception. N Engl J Med 2012;366(21):1998-200 